\title{
Drink, drugs and disruption: memory manipulation for the treatment of addiction
}

\author{
A.L. Milton \\ Behavioural and Clinical Neuroscience Institute, Department of Psychology, University of \\ Cambridge, Downing Site, Cambridge CB2 3EB, UK. \\ Word counts \\ Abstract: 127 words \\ Total: 2387 words \\ Pages: 19 \\ Figures: 2 \\ Corresponding author: \\ Dr Amy L. Milton \\ Behavioural and Clinical Neuroscience Institute \\ Department of Psychology \\ University of Cambridge \\ Downing Site \\ Cambridge \\ CB2 3EB \\ UK \\ Tel: +44 1223333593 \\ Fax: +44 1223333564 \\ Email: alm46@cam.ac.uk
}




\section{ABstract}

Addiction is a complex disorder, and one characterised by the acquisition of maladaptive instrumental (drug-seeking and drug-taking) and pavlovian (cue-drug associations) memories. These memories markedly contribute to the long-term risk of relapse, so reduction of the impact of these memories on behaviour could potentially be an important addition to current therapies for addiction. Memory reconsolidation may provide such a target for disrupting well-consolidated pavlovian cue-drug memories following an extensive drug history. Reconsolidation can be disrupted either by administering amnestic drugs in conjunction with a memory reactivation session, or by updating the memory adaptively through the induction of 'superextinction'. More work is needed before these therapies are ready for translation to the clinic, but if found clinically effective memory manipulation promises a radical new way of treating addiction. 


\section{INTRODUCTION}

Addiction is a chronic disorder associated with a long-term risk of relapse. Although complex, one increasingly dominant view in the addiction literature conceptualises the disease as one of aberrant learning and memory $[1,2]$. For this reason, there has been much interest in manipulating the maladaptive memories that underlie the long-term risk of relapse, by targeting the process of memory reconsolidation. This review will outline how maladaptive memories contribute to relapse risk in addiction, before going on to describe reconsolidation and how it might be used to disrupt drug memories. Finally, the feasibility of disrupting drug memories will be considered, by discussing how research conducted in animal models might be translated to a clinical setting.

\section{ADDICTION AS A DISORDER OF MALADAPTIVE LEARNING AND MEMORY}

Addiction can be conceptualised as a disorder of learning and memory [1,2]; the acts of drug-seeking and drug-taking are learned instrumental associations that occur in the presence of initially neutral environmental cues that become associated with the drug high through the process of pavlovian conditioning (Figure 1). These pavlovian conditioned stimuli (CSs) come to influence ongoing drug-seeking behaviour, and precipitate relapse in individuals trying to remain abstinent [3].

Pavlovian memories influence drug-seeking and relapse behaviour through at least three psychologically and neurobiologically distinct processes [4; Figure 1]. These processes - conditioned reinforcement, conditioned motivation and conditioned approach - support drug-seeking over delays to reinforcement, enhance ongoing drug-seeking, and bring the individual into the vicinity of where the drug-seeking response can be made [5] respectively. Thus, although relapse to drug-seeking is instrumental, it is influenced markedly by pavlovian associations that unconsciously influence behaviour. 
If maladaptive CS-drug memories contribute to maintaining drug-seeking and precipitating relapse, then manipulating these memories should reduce their effects on behaviour. Cue-exposure therapy, in which individuals are re-exposed to drug-associated stimuli in the absence of the drug high, aims to achieve these through 'extinction' (Figure 2c). However, rather than 'unlearning' the original CS-US association, extinction instead involves the learning of a new, 'CS-no US' association [6]. Subsequently, the two associations compete for behavioural expression, with the original CS-US association being less contextually bound than the extinction memory. Thus, with a change in time, context or internal state through re-exposure to the US, the original CS-US memory can come to dominate behaviour again by spontaneous recovery, renewal or reinstatement [7]. Cueexposure therapy has had limited success in treating addiction, and although it may be possible to pharmacologically enhance the efficacy of extinction-based therapies $[8,9]$, perhaps more effective would be strategies based upon manipulating the CS-drug memory itself.

\section{MEMORY MANIPULATION AS A POTENTIAL STRATEGY FOR TREATING ADDICTION}

The CS-drug memories underlying addiction are acquired through an extensive selfadministration history, and so are well-consolidated when patients present for treatment. In this situation, reconsolidation is a more feasible target for disruption than consolidation. Reconsolidation (Figure $\mathbf{2 a}$ ) is the process by which previously consolidated memories are updated and maintained in the brain [10], following their reactivation (typically induced through memory retrieval in the laboratory). Following the initial demonstration of reconsolidation in the late 1960s [11,12], the process was little studied until the turn of the century [13]. However, with recognition of the importance of maladaptive memories in neuropsychiatric disorders, an increasing amount is becoming known about the molecular 
and neurochemical mechanisms that underlie the reconsolidation process, and the boundary conditions that constrain it.

\section{THE DISRUPTION OF DRUG-MEMORY RECONSOLIDATION IN ANIMAL MODELS}

Animal models of addiction vary in their translational relevance, from procedures that use limited pairings of CSs and experimenter-administered drugs (e.g. conditioned place preference, or CPP) to behavioural tasks that involve hundreds of pairings of CSs with selfadministered drugs of abuse. Regardless of the model used, many studies have found that reconsolidation depends upon neurochemical signalling that is hypothesised to lead to increased expression of the plasticity-related immediate early gene zif268, and ultimately protein synthesis.

\section{PROTEIN SYNTHESIS AND INTRACELLULAR SIGNALLING CASCADES}

Consistent with previous work on fear memory reconsolidation [13], the reconsolidation of CS-drug memories is known to depend upon protein synthesis. Protein synthesis inhibition, in conjunction with memory reactivation, not only reduces subsequent preference for a previously cocaine-paired side in a CPP procedure $[14,15]$ but also prevents a cocaineassociated CS from acting subsequently as a conditioned reinforcer [16]. This mechanism is not specific to cocaine as a reinforcer, however, since protein synthesis inhibition at reactivation also disrupts CS-alcohol memories [17].

The proteins required for memory restabilisation are regulated by the expression of the plasticity-related immediate early gene zif268, which is critical for reconsolidation to occur [18]. Selective impairment of Zif268 protein expression using targeted antisense oligodeoxynucleotides prior to reactivation not only disrupted the reconsolidation of the memory underlying cocaine-conditioned place preference [19], but also prevented a cocaine-associated CS from subsequently acting as a conditioned reinforcer, similar to the effect seen with protein synthesis inhibition $[16,19]$ and reduced the impact of a cocaine- 
associated CS on relapse to drug-seeking behaviour [20]. Protein synthesis, driven by Zif268 expression, therefore appears critical for the reconsolidation of CS-drug memories.

The dual regulation of zif268 by both the cyclic AMP response element (CRE) and the serum response element (SRE) indicates that a number of intracellular signalling cascades can potentially activate its expression, though to date there have been few studies directly linking inhibition of specific pathways to the reduction of Zif268. It is known, for instance, that administration of a PKA inhibitor can prevent the CS-induced reinstatement of cocaineseeking [21] and that inhibition of extracellular-signal regulated kinase (ERK) prevents the reconsolidation of the memories underlying cocaine-conditioned place preference [15], but Zif268 levels were not assessed in these studies. The requirement for these intracellular protein kinases is also suggestive as to the neurochemical mechanisms of reconsolidation.

\section{NEUROCHEMICAL MECHANISMS}

Most studies investigating the neurochemical mechanisms underlying memory reconsolidation have focused on either $\beta$-adrenergic receptors ( $\beta A R s$ ) or the NMDA subtype of glutamate receptor (NMDARs). Both neurochemical systems have been linked to memory consolidation [22,23] and synaptic plasticity [24], and are required for the reconsolidation of pavlovian CS-fear memories [25,26]. Furthermore, there has been great interest in $\beta A R-$ mediated signalling because of the therapeutic potential of the $\beta A R$ antagonist propranolol, which is already approved for human use.

Propranolol is effective at reducing, if not fully disrupting, the reconsolidation of CSdrug memories. Propranolol disrupts the reconsolidation of the memories underlying cocaine-conditioned place preference [27], most likely by acting on central $\beta_{2}$-adrenergic receptors [28]. However, the effects of propranolol on reconsolidation have been variable, particularly with regard to the number of treatment/reactivation sessions required for amnesia; single sessions are sufficient in some laboratories [27,28] but not others [29]. 
Sometimes, for instance when targeting reconsolidation of the memories underlying a place preference conditioned to alcohol, propranolol does not appear to be effective at all [30]. These apparent inconsistencies may, in fact, reflect differences in the associations supporting the behaviour, in procedures that may differ slightly from one laboratory to another.

Self-administration models can be informative in this respect. Propranolol, given at reactivation, has been shown to reduce the capacity of a previously cocaine-associated CS to act subsequently as a conditioned reinforcer [31]. However, surprisingly, the same dose given to animals with a similar CS-drug history was ineffective at reducing CS-induced reinstatement of cocaine-seeking [32]. (Though it should be noted that propranolol does reduce the reinstatement of CS-induced alcohol-seeking when it is given in multiple treatment/reactivation sessions [33].) Analysis of CS-induced reinstatement procedures suggests that the effects of the drug-associated CS on instrumental behaviour could, in fact, be mediated by any one (or a combination) of the processes of conditioned reinforcement, conditioned motivation, or conditioned approach (Figure 1). Disruption of, for instance, the conditioned reinforcement memory alone may not reduce CS-induced relapse behaviour if conditioned motivation and approach could still act to support it [4]. It is relevant, therefore, that propranolol, given prior to reactivation, does not disrupt the reconsolidation of the CSalcohol memories that support conditioned motivation and conditioned approach [34]. A similar argument could be made for the apparently inconsistent findings with CPP procedures, since there has not been a detailed psychological or neurobiological analysis of the memories that support place preference, nor how much one association could potentially behaviourally compensate for another, disrupted one.

The dependence of CS-drug memory reconsolidation on NMDAR-mediated signalling has been more consistent. The NMDAR antagonist MK-801 has been shown to disrupt the 
reconsolidation of the memories underlying cocaine CPP [35] and CS-induced relapse for cocaine $[36]$ and alcohol $[17,33]$. An interesting point, from the perspective of the previous discussion, is that NMDAR antagonism at reactivation is effective at disrupting the memories that underlie conditioned motivation and conditioned approach for an alcohol-associated CS [34]. This may explain the different effects of NMDAR antagonism and $\beta A R$ antagonism on CS-induced reinstatement [32]; as NMDAR antagonism can disrupt the reconsolidation of all 'three routes to relapse', then after treatment, no pavlovian associations remain intact to influence instrumental behaviour. This is not the case with $\beta A R$ antagonism, where only the memory underlying conditioned reinforcement is sensitive to disruption so, as suggested above, conditioned approach and conditioned motivation continue to support the influence of pavlovian CSs on relapse behaviour.

\section{BEHAVIOURAL THERAPIES: 'SUPEREXTINCTION'}

The disruption of CS-drug memories underlying relapse to drug-seeking behaviour may be a viable therapeutic strategy in the treatment of addiction. However, the development of pharmacotherapies appropriate for human use is not trivial, and although NMDAR antagonists have proven highly effective in animal studies, the psychotomimetic side effects of these drugs makes translation to clinical use difficult. An alternative 'disruption' strategy that has received much interest is the phenomenon of 'extinction within the reconsolidation window', or 'superextinction'. Originally observed in studies of conditioned fear [37], though not universally replicated [38], superextinction refers to the updating of the original CS-US memory to a CS-no US association, rather than the learning of a separate, competing CS-no US memory, as in extinction (cf. Figure $\mathbf{2 c}$ and Figure $\mathbf{2 d}$ ). Briefly, superextinction is induced by first initiating memory destabilisation through reactivation, similar to studies using pharmacological manipulations to disrupt reconsolidation. Following a short break in which the animal is removed from the reactivation context - typically lasting 10 minutes to 1 hour - the animal is returned to the same context to complete extinction training (Figure 2d) so 
updating the old memory (with the information that the CS is now 'safe' in the case of CSfear memories). Pharmacological prevention of destabilisation has been shown to prevent the modulation of memory strength [39], and it is hypothesised that the introduction of the delay between the reactivation and extinction training phases alters the dynamics of the switch between the reconsolidation and extinction processes [40].

The only demonstration to date of superextinction as a means of disrupting CS-drug memories is work by Xue and colleagues [41], who superextinguished the memories underlying both conditioned place preference and CS-induced relapse in animals previously trained to self-administer cocaine or heroin. Most remarkably of all, the superextinction treatment was also effective in preventing relapse for 6 months in human heroin addicts trying to maintain abstinence, when the investigators adapted standard cue-exposure therapy to incorporate the break between memory reactivation and extinction training. Though this study requires replication and further long-term follow-up, these findings are extremely encouraging for developing treatments for addiction based on manipulating maladaptive memories.

\section{TRANSLATION OF MEMORY MANIPULATION THERAPIES TO THE CLINIC}

Of course, the clinical translation of memory-manipulation treatments is not trivial, and further research is needed before treatments based on the manipulation of memories could be made widely available. One issue, previously alluded to, is the development of an appropriate amnestic agent if attempting to disrupt memory reconsolidation pharmacologically. Further research into the neurochemical systems underlying memory reconsolidation is needed to identify new drug targets, or more selective receptor antagonists could be developed by the pharmaceutical industry. Alternatively, this issue could be bypassed if the behavioural therapy of superextinction is consistently found to be effective. 
Another important consideration, for both pharmacological and behavioural manipulations of memory, is the understanding of boundary conditions, particularly between reconsolidation and extinction. A parametric study of memory reactivation is still lacking in the literature. In my laboratory, we have repeatedly found that behaviourally contingent reactivation sessions consisting of approximately $5-10 \%$ of total training exposures are effective in reactivating appetitive memories for cocaine, alcohol and sucrose, though it is not clear that these parameters are optimal. Understanding CS-drug memory reactivation and its relation to extinction is especially important, since pharmacological treatments that act to disrupt memory reconsolidation also often act to disrupt extinction, subsequently with opposite behavioural effects [26]. This is a pertinent issue for cueexposure therapy, since CS-drug memories are highly resistant to extinction [42]. Indeed, an attempt to use the NMDAR partial agonist D-cycloserine to enhance the extinction of cocaine-associated memories in a patient population actually increased subsequent craving for cocaine [43], consistent with enhancement of CS-cocaine memory reconsolidation dependent on Zif268 [44], rather than enhancement of extinction.

One final, but important, point to consider is the specificity of memory disruption. Though previous work has demonstrated relatively selective disruption of memory reconsolidation with pharmacological interventions $[45,46]$, a recent study suggests that amnesia may not be selective when animals are trained on multiple tasks dependent upon the same brain structure. When mice were trained on both cocaine-conditioned place preference and an inhibitory avoidance task, it was found that the inactivation of the basolateral amygdala following reactivation of either the cocaine-place memory or the shock-place memory was sufficient to impair performance on both tasks [47]. It is not yet clear whether this finding is specific to the tasks used, or is indicative of a spreading amnesia when a neural locus required for more than one type of memory is targeted. This is especially relevant in the context of recent work suggesting that retrieval may not be a 
necessary condition for memory destabilisation and reconsolidation; the processes of memory retrieval, destabilisation and restabilisation are, in fact, dissociable, at least for pavlovian conditioned fear memories [48]. Further research is required to determine how extensively memories are disrupted when a specific memory is targeted.

\section{CONCLUSIONS}

Addiction is a complex psychiatric disorder, and although maladaptive memories are only one aspect of addiction, they are extremely difficult to treat. Advances in understanding the mechanisms by which memories persist have identified that manipulation of drug memories may provide a promising therapeutic strategy for addiction, though more work is needed before this therapy could be implemented on a wide scale.

\section{ACKNOWLEDGEMENTS}

This work was supported by a UK Medical Research Council Programme Grant (no. 1002231) and was conducted in the Behavioural and Clinical Neuroscience Institute, funded by a joint award from the Medical Research Council and the Wellcome Trust. 


\section{References}

1. Everitt BJ, Dickinson A, Robbins TW: The neuropsychological basis of addictive behaviour. Brain Res Rev (2001) 36:129-138.

2. Everitt BJ, Robbins TW: Neural systems of reinforcement for drug addiction: from actions to habits to compulsion. Nat Neurosci (2005) 8:1481-1489.

3. de Wit H, Stewart J: Reinstatement of cocaine-reinforced responding in the rat. Psychopharmacology (1981) 75:134-143.

4. Milton AL, Everitt BJ: The psychological and neurochemical mechanisms of drug memory reconsolidation: implications for the treatment of addiction. Eur J Neurosci (2010) 31:2308-2319.

5. Tomie A: CAM: an animal learning model of excessive and compulsive implementassisted drug-taking in humans. In: Clinical Psychology Review. 15. (1995):145-167.

6. Bouton ME, Bolles RC: Contextual control of the extinction of conditioned fear. Learn Motiv (1979) 10:445-466.

7. Bouton ME: Context, ambiguity, and unlearning: sources of relapse after behavioral extinction. Biological Psychiatry (2002) 52:976-986.

8. Nic Dhonnchadha BA, Kantak KM: Cognitive enhancers for facilitating drug cue extinction: insights from animal models. Pharmacology, Biochemistry, and Behavior (2011) 99:229-244.

9. Torregrossa MM, Sanchez H, Taylor JR: D-cycloserine reduces the context specificity of pavlovian extinction of cocaine cues through actions in the nucleus accumbens. The Journal of Neuroscience (2010) 30:10526-10533.

10. Lee JLC: Reconsolidation: maintaining memory relevance. Trends Neurosci (2009) 32:413-420.

11. Misanin JR, Miller RR, Lewis DJ: Retrograde amnesia produced by electroconvulsive shock after reactivation of a consolidated memory trace. Science (1968) 160:554555 .

12. Schneider AM, Sherman W: Amnesia: a function of the temporal relation of footshock to electroconvulsive shock. Science (1968) 159:219-222. 
13. Nader K, Schafe GE, LeDoux JE: Fear memories require protein synthesis in the amygdala for reconsolidation after retrieval. Nature (2000) 406:722-726.

14. Bernardi RE, Lattal KM, Berger SP: Anisomycin disrupts a contextual memory following reactivation in a cocaine-induced locomotor activity paradigm. Behav Neurosci (2007) 121:156-163.

15. Valjent E, Corbillé AG, Bertran-Gonzelez J, Hervé D, Girault JA: Inhibition of ERK pathway or protein synthesis during reexposure to drugs of abuse erases previously learned place preference. Proceedings of the National Academy of Sciences (2006) 103:2932-2937.

16. Lee JLC, Di Ciano P, Thomas KL, Everitt BJ: Disrupting reconsolidation of drug memories reduces cocaine seeking behavior. Neuron (2005) 47:795-801.

17. von der Goltz C, Vengeliene V, Bilbao A, Perreau-Lenz S, Pawlak CR, Kiefer F, Spanagel R: Cue-induced alcohol seeking behaviour is reduced by disrupting the reconsolidation of alcohol-related memories. Psychopharmacology (2009) 205:389397.

18. Lee JLC, Everitt BJ, Thomas KL: Independent cellular processes for hippocampal memory consolidation and reconsolidation. Science (2004) 304:839-843.

19. Théberge FR, Milton AL, Belin D, Lee JLC, Everitt BJ: The basolateral amygdala and nucleus accumbens core mediate dissociable aspects of drug memory reconsolidation. Learn Memory (2010) 17:444-453.

20. Lee JLC, Milton AL, Everitt BJ: Cue-induced cocaine seeking and relapse are reduced by disruption of drug memory reconsolidation. The Journal of Neuroscience (2006) 26:5881-5887.

21. Sanchez H, Quinn JJ, Torregrossa MM, Taylor JR: Reconsolidation of a cocaineassociated stimulus requires amygdalar protein kinase $\mathbf{A}$. The Journal of Neuroscience (2010) 30:4401-4407.

22. Morris RG, Anderson E, Lynch GS, Baudry M: Selective impairment of learning and blockade of long-term potentiation by an $\mathbf{N}$-methyl-D-aspartate receptor antagonist, AP5. In: Nature. 319. (1986):774-776.

23. Wilson DA, Pham TC, Sullivan RM: Norepinephrine and posttraining memory consolidation in neonatal rats. In: Behavioral Neuroscience. 108. (1994):1053-1058. 
24. Collingridge GL, Kehl SJ, McLennan H: Excitatory amino acids in synaptic transmission in the Schaffer collateral-commissural pathway of the rat hippocampus. The Journal of Physiology (1983) 334:33-46.

25. Dębiec J, LeDoux JE: Disruption of reconsolidation but not consolidation of auditory fear conditioning by noradrenergic blockade in the amygdala. Neuroscience (2004) 129:267-272.

26. Lee JLC, Milton AL, Everitt BJ: Reconsolidation and extinction of conditioned fear: inhibition and potentiation. The Journal of Neuroscience (2006) 26:10051-10056.

27. Bernardi RE, Lattal KM, Berger SP: Postretrieval propranolol disrupts a cocaine conditioned place preference. Neuroreport (2006) 17:1443-1447.

28. Bernardi RE, Ryabinin AE, Berger SP, Lattal KM: Post-retrieval disruption of a cocaine conditioned place preference by systemic and intrabasolateral amygdala $\beta_{2}$ and $\alpha_{1}$-adrenergic antagonists. Learn Memory (2009) 16:777-789.

29. Fricks-Gleason AN, Marshall JF: Post-retrieval $\beta$-adrenergic receptor blockade: effects on extinction and reconsolidation of cocaine-cue memories. Learn Memory (2008) 15:643-648.

30. Font L, Cunningham CL: Post-retrieval propranolol treatment does not modulate reconsolidation or extinction of ethanol-induced conditioned place preference. Pharmacol Biochem Behav (2012) 101:222-230.

31. Milton AL, Lee JLC, Everitt BJ: Reconsolidation of appetitive memories for both natural and drug reinforcement is dependent on $\beta$-adrenergic receptors. Learn Memory (2008) 15:88-92.

32. Milton AL, Everitt BJ: NMDA receptors and beta-adrenergic receptors as molecular targets for the prevention of relapse to drug-seeking. European Neuropsychopharmacology (2009) 19:S86-S87.

33. Wouda JA, Diergaarde L, Riga D, Van Mourik Y, Schoffelmeer ANM, De Vries TJ: Disruption of long-term alcohol-related memory reconsolidation: role of $\beta$ adrenoceptors and NMDA receptors. Frontiers in Behavioral Neuroscience (2010) 4:179. doi: 110.3389/fnbeh.2010.00179.

34. Milton AL, Schramm MJW, Wawrzynski J, Gore F, Oikonomou-Mpegeti F, Wang NQ, Samuel D, Economidou D, Everitt BJ: Antagonism at NMDA receptors, but not $\beta$ adrenergic receptors, disrupts the reconsolidation of pavlovian conditioned 
approach and instrumental transfer for ethanol-associated conditioned stimuli. Psychopharmacology (2012) 219:751-761.

35. Itzhak Y: Role of the NMDA receptor and nitric oxide in memory reconsolidation of cocaine-induced conditioned place preference in mice. Ann N Y Acad Sci (2008) 1139:350-357.

36. Milton AL, Lee JLC, Butler VJ, Gardner RJ, Everitt BJ: Intra-amygdala and systemic antagonism of NMDA receptors prevents the reconsolidation of drug-associated memory and impairs subsequently both novel and previously acquired drugseeking behaviors. The Journal of Neuroscience (2008) 28:8230-8237.

37. Monfils MH, Cowansage KK, Klann E, LeDoux JE: Extinction-reconsolidation boundaries: key to persistent attenuation of fear memories. Science (2009) 324:951-955.

38. Chan WY, Leung HT, Westbrook RF, McNally GP: Effects of recent exposure to a conditioned stimulus on extinction of pavlovian fear conditioning. Learn Memory (2010) 17:512-521.

39. Lee SH, Choi JH, Lee N, Lee HR, Kim JI, Yu NK, Choi SL, Kim H, Kaang BK: Synaptic protein degradation underlies destabilization of retrieved fear memory. Science (2008) 319:1253-1256.

40. Merlo E, Goozée Z, Milton AL, Everitt BJ: NMDAR-dependent increase in calcineurin in the basolateral amygdala governs the transition from reconsolidation to extinction of fear memory. Society for Neuroscience, New Orleans, LA (2012):Abs Programme No. 291.206.

41. Xue Y-X, Luo Y-X, Wu P, Shi H-S, Xue L-F, Chen C, Zhu W-L, Ding Z-B, Bao Y-P, Shi J, Epstein $\mathrm{DH}$ et al: A memory retrieval-extinction procedure to prevent drug craving and relapse. Science (2012) 336:241-245.

42. Di Ciano P, Everitt BJ: Conditioned reinforcing properties of stimuli paired with selfadminstered cocaine, heroin or sucrose: implications for the persistence of addictive behavior. Neuropharmacology (2004) 47:202-213.

43. Price KL, McRae-Clark AL, Saladin ME, Maria MM, DeSantis SM, Back SE, Brady KT: Dcycloserine and cocaine cue reactivity: preliminary findings. . The American Journal of Drug and Alcohol Abuse (2009) 35:434-438.

44. Lee JLC, Gardner RJ, Butler VJ, Everitt BJ: D-cycloserine potentiates the reconsolidation of cocaine-associated memories. Learn Memory (2009) 16:82-85. 
45. Dębiec J, Doyere V, Nader K, LeDoux JE: Directly reactivated, but not indirectly reactivated, memories undergo reconsolidation in the amygdala. Proceedings of the National Academy of Sciences (2006) 103:3428-3433.

46. Doyère V, Dębiec J, Monfils MH, Schafe GE, LeDoux JE: Synapse-specific reconsolidation of distinct fear memories in the lateral amygdala. (2007) 10:414416.

47. Tzeng W-Y, Chang W-T, Chuang J-Y, Lin K-Y, Cherng CG, Yu L: Disruption of memory reconsolidation impairs storage of other, non-reactivated memory. Neurobiol Learn Mem (2012) 97:241-249.

48. Milton AL, Merlo E, Ratano P, Gregory BL, Dumbreck JK, Everitt BJ: Double dissociation of the requirement for GluN2B- and GluN2A-containing NMDA receptors in the destabilization and restabilization of a reconsolidating memory. The Journal of Neuroscience (In press.).

\section{FIGURE LEGENDS}

Figure 1. Pavlovian CS-drug memories influence ongoing instrumental behaviour to maintain drug-seeking behaviour and to precipitate relapse. Individuals learn to make drug-seeking and drug-taking responses initially because the action is associated with the action of a drug high (though ultimately these responses can become divorced from the outcome, and so habitual). These instrumental memories are acquired in the presence of environmental stimuli that become associated with the drug in a pavlovian manner, and can influence instrumental behaviour by supporting approach towards the stimulus and where the instrumental response can be made (conditioned approach); by enhancing the motivation to make an instrumental response for the outcome (conditioned motivation, or pavlovianinstrumental transfer) or by supporting responding over delays to reinforcement (conditioned reinforcement). The reconsolidation of the memories underlying these conditioned approach and conditioned motivation can be disrupted by antagonism at 
NMDARs (red boxes) while that underlying conditioned reinforcement can be disrupted by antagonism at either NMDARs or $\beta A R s$ (red/blue box).

Figure 2. Mechanisms of memory manipulation. (a) Reconsolidation is the process by which a previously consolidated memory in the 'inactive state' is converted (reactivated) to the 'active state' through destabilisation mechanisms (normally induced in the laboratory by memory retrieval). These 'active' memories are subsequently restabilised back into the 'inactive' state in a protein-synthesis dependent manner. (b) Disruption of reconsolidation (restabilisation) offers a therapeutic strategy for reducing the impact of maladaptive memories on behaviour. By combining memory reactivation (red box) with the administration of an amnestic agent (red 'no' sign), reconsolidation is blocked and the memory degrades from the unstable 'active' state. (c) Extinction does not require conversion between the 'inactive' and 'active' states of a memory, but instead is the learning of a new CS-no US memory. The two memories both persist in the brain and compete for behavioural expression, inhibiting each other. (d) 'Superextinction' exploits the reconsolidation process to rewrite old memories. By inducing memory reactivation (red box) and then, with an appropriate delay, introducing new information (red arrow), the memory is reconsolidated to persist in the 'inactive state' in the modified, updated form. 


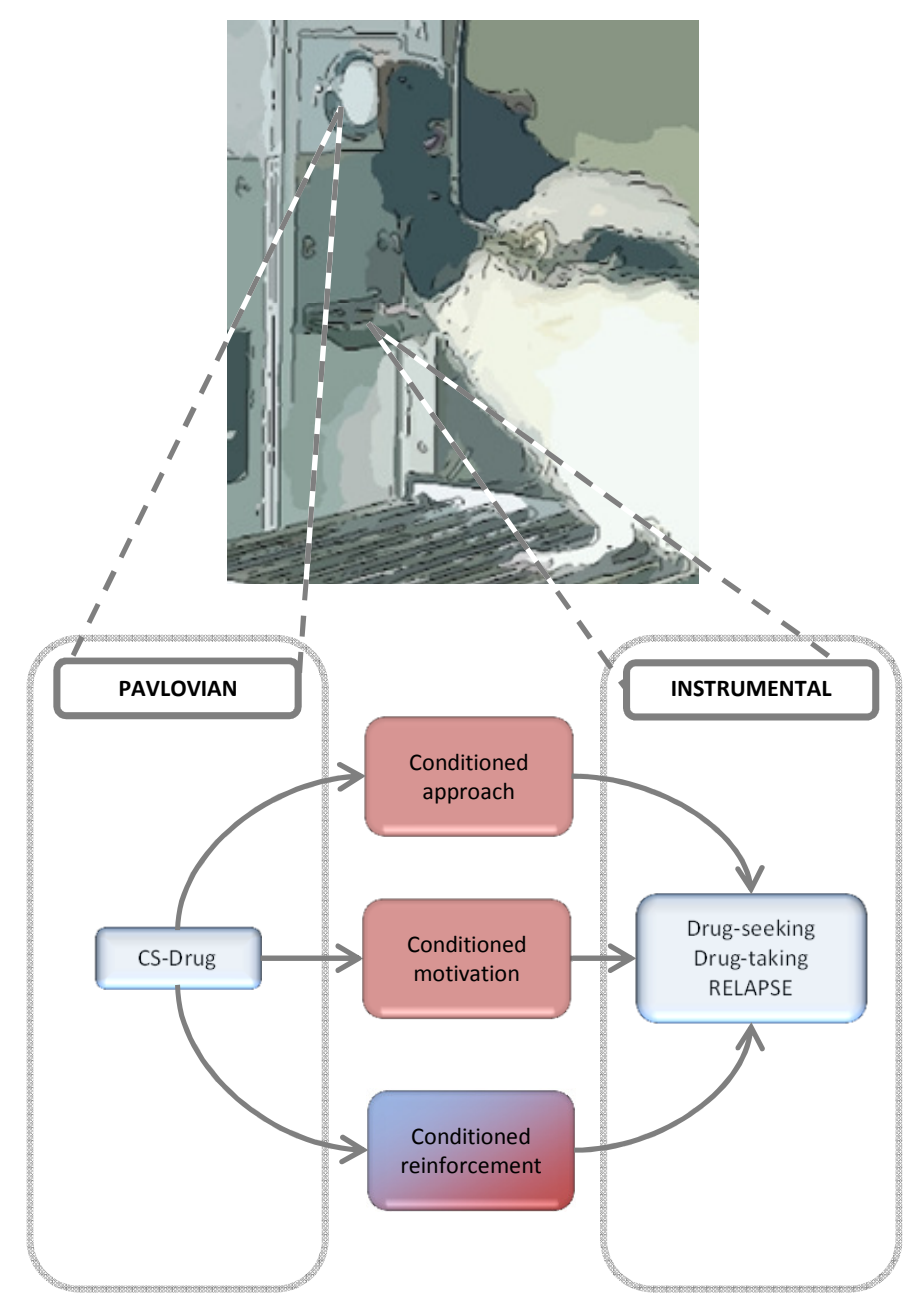


(a)

Reconsolidation / restabilisation via protein synthesis, Zif expression

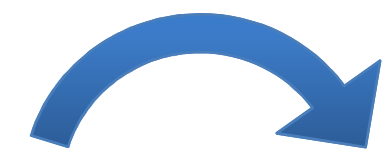

Active state

- Transient

Inactive state

- Susceptible

- Persistent

to disruption

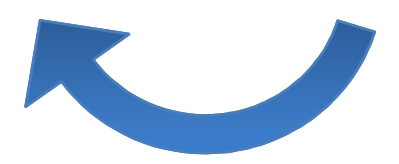

Reactivation / destabilisation via protein degradation; usually induced through retrieval

(c)

Original CS-US memory (inactive)

$$
0
$$

New CS- no US memory (inactive) (b)

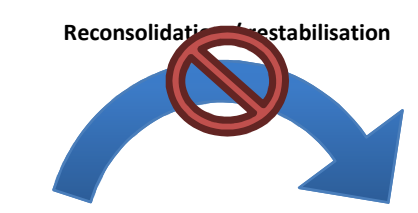

Active state

Inactive state

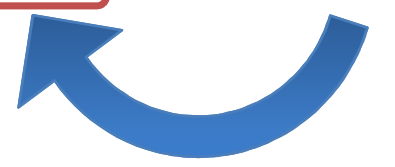

Reactivation / destabilisation

(d)

Reconsolidation / restabilisation New informatio
(CS-no US)

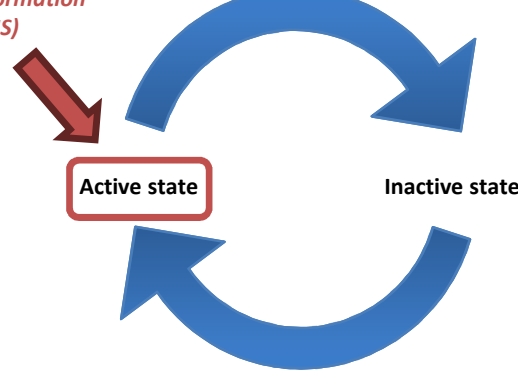

Reactivation / destabilisation 